\title{
Unusual polarization ordering in incommensurate phases on the ferroelectric - antiferroelectric boundary in lanthanum doped lead zirconate titanate
}

\author{
R. Villaurrutia*, I. MacLaren*, A. Pelaiz-Barranco** \\ * Department of Physics and Astronomy, University of Glasgow, Glasgow G12 8QQ, U.K. \\ ** Facultad de Física/IMRE, San Lázaro y L, Univ. de la Habana, C. Habana 10400, Cuba
}

Lanthanum doped Lead Zirconate Titanate (PLZT) $\mathrm{PbLa}_{\mathrm{x}} \mathrm{Zr}_{1-\mathrm{y}} \mathrm{Ti}_{\mathrm{y}} \mathrm{O}_{3}$ is an exciting functional material with very important technological applications in the electronic industry. Of particular scientific interest is the phase transition from a rhombohedral ferroelectric (FE) to an orthorhombic anti-ferroelectric (AFE) phase. In undoped material this occurs at about $y=5 \%$, but the FE-AFE transition also happens with La doping at $\mathrm{y}=10 \%$ and this seems to happen between the 2:90:10 and 3:90:10 compositions. In order to understand the behaviour and properties of such materials a detailed study was performed using transmission electron microscopy.

The present work concentrates on a detailed study of the micro and nano-sized domain structures found in 2:90:10, 3:90:10 and 4:90:10 compositions using dark field imaging, recent advances in automated analysis of Kikuchi diffraction patterns, conventional selected area diffraction, and in-situ measurements at high and low temperature. Figure 1 shows images of one grain recorded under a variety of diffraction conditions. Under bright field illumination and in strong beam dark field conditions, the dominant contrast is typical TEM contrast of bend contours and domain boundaries, as exemplified by the image of Figure 1a. Whilst individual domain boundaries are rarely straight, and wedge shaped domains are common, most domain boundaries show a strong tendency towards following $\{101\}$ planes. Within these domains, a weaker nanostructure can be observed in some very weak diffraction conditions (e.g. Figures 1b-d). In the inset in Figure 1d, weak satellite spots can be seen running in a direction perpendicular to the streaking of the nanostructure. These run in $<110>$ directions and correspond to a large d-spacing which has been measured as about $26 \pm 2 \AA$ and $23 \pm 2 \AA$ for two such diffraction patterns, corresponding to about 8 or 9 (110) spacing; these clearly correspond to those previously seen by Dai et $a l^{2}$ and must arise from a long-period ordered but incommensurate structure and are clearly inconsistent with a rhombohedral PZT structure. Further analysis of the allowed and forbidden reflections in diffraction patterns shows that this phase is an incommensurate version of the AFE $\mathrm{PbZrO}_{3}$ phase. Using Kikuchi patterns, it is possible to measure the relative orientations between the domains, as was done for the area of a 4:90:10 composition shown in Figure 2, and these misorientations also correspond very well to expectations on the assumption that the lattice parameters are close to those for $\mathrm{PbZrO}_{3}$ and also allow us to make good models of the structure of the domains and their internal nanostructures. The interesting feature is that such incommensurate phases are present on both sides of the FE-AFE boundary, and this is an unusual case of a FE phase perovskite with a polarization axis in the $<110>$ direction.

References

[1] M.U. Farooq, R. Villaurrutia, I. MacLaren, H. Kungl, M.J. Hoffmann, J.-J. Fundenberger, and E. Bouzy, Journal of Microscopy, 230, 445 (2008).

[2] X.H. Dai, Z. Xu, J.F. Li, and D. Viehland, Journal of Materials Research, 11, 626 (1996).

[3] P.S. Bate, R.D. Knutsen, I. Brough, and F.J. Humphreys, Journal of Microscopy. (Oxford), 220, 36 (2005). 


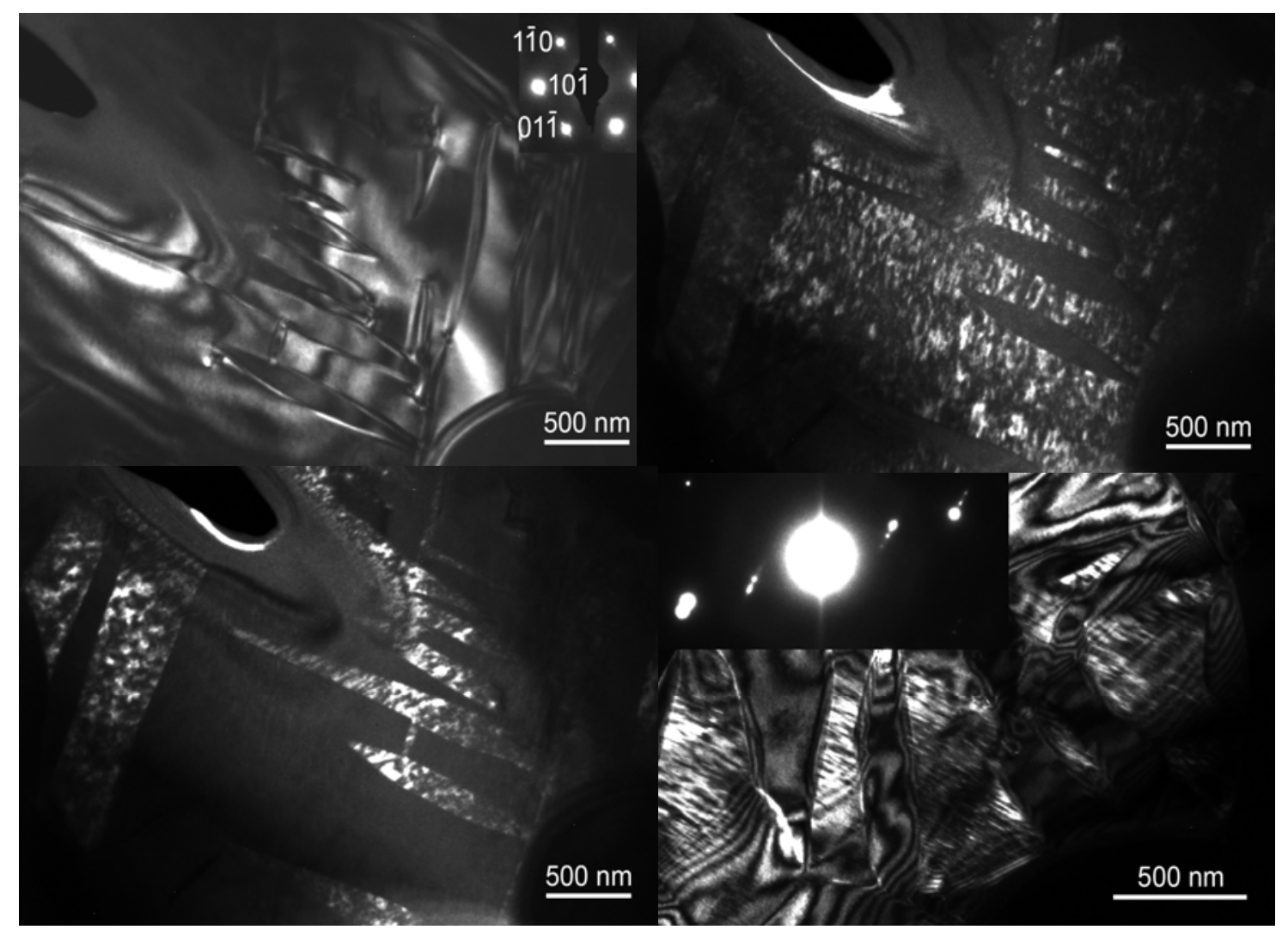

FIG. 1 Dark field TEM of the domain structure in a 2:90:10 specimen; a) Domain contrast only; b) Nanostructure in one set of domains; c) Nanostructure in a complementary set of domains; d) Relationship between the nanostructure and the satellite spots.

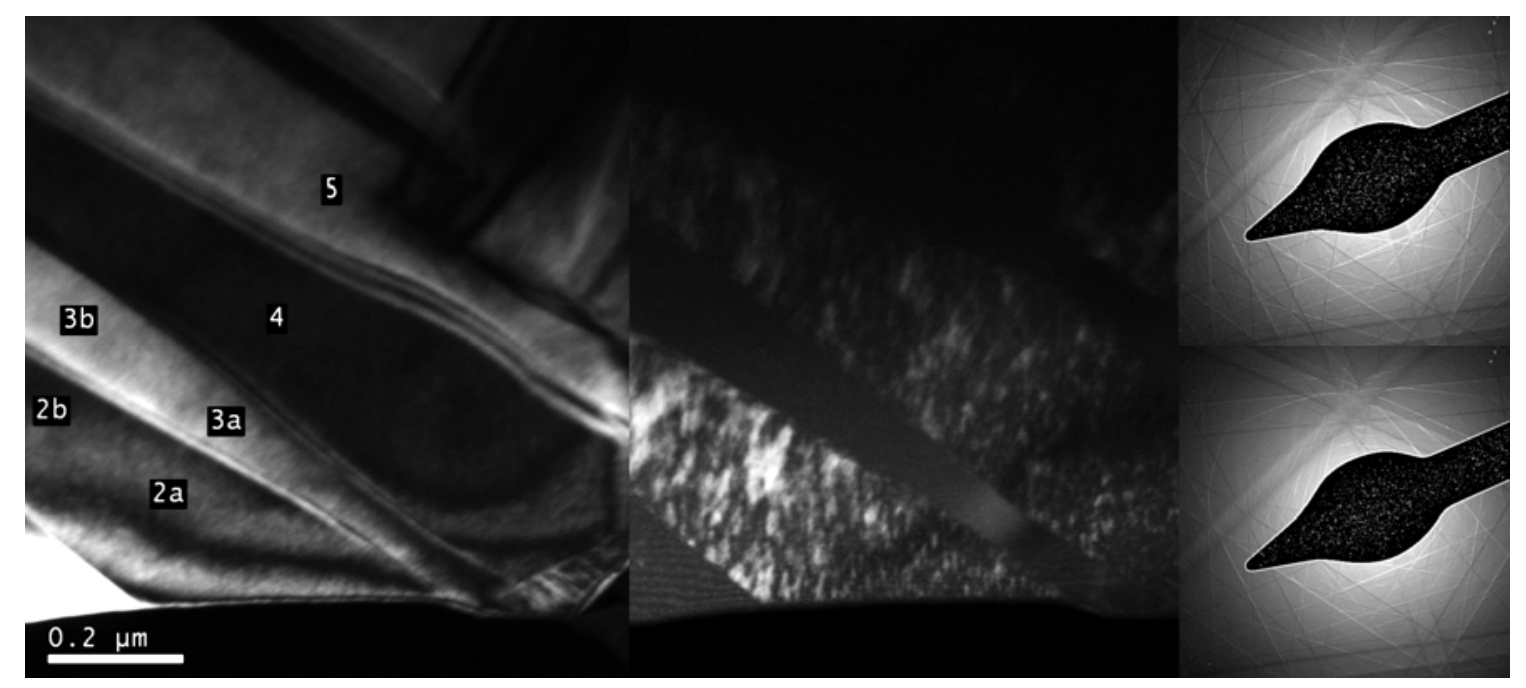

FIG. 2 Structures in a PLZT 4:90:10 sample: a) Strong beam dark field image of the domain structure; b) weaker beam image of the nanostructure in domains 2 and 4; c) processed Kikuchi pattern from point $3 \mathrm{a}$; d) processed Kikuchi pattern from point 4 . 